\title{
Global Predicate Detection under Fine-Grained Modalities
}

\author{
Punit Chandra and Ajay D. Kshemkalyani \\ Computer Science Department, \\ Univ. of Illinois at Chicago, Chicago, IL 60607, USA \\ \{pchandra, ajayk\}@cs.uic.edu
}

\begin{abstract}
Predicate detection is an important problem in distributed systems. Based on the temporal interactions of intervals, there exists a rich class of modalities under which global predicates can be specified. For a conjunctive predicate $\phi$, we show how to detect the traditional Possibly $(\phi)$ and Definitely $(\phi)$ modalities along with the added information of the exact interaction type between each pair of intervals (one interval at each process). The polynomial time, space, and message complexities of the proposed on-line detection algorithms to detect Possibly and Definitely in terms of the fine-grained interaction types per pair of processes, are the same as those of the earlier on-line algorithms that can detect only whether the Possibly and Definitely modalities hold.
\end{abstract}

\section{Introduction}

Predicate detection in a distributed system is useful in many contexts such as monitoring, synchronization and coordination, debugging, and industrial process control 2 24678 14 16 17. Marzullo et al. defined two modalities under which predicates can hold for a distributed execution 4,14.

- Possibly $(\phi)$ : There exists a consistent observation of the execution such that $\phi$ holds in a global state of the observation.

- Definitely $(\phi)$ : For every consistent observation of the execution, there exists a global state of it in which $\phi$ holds.

The formalism and axiom system given in [9] identified an orthogonal set $\Re$ of 40 fine-grained temporal interactions between a pair of intervals in a distributed execution. It was shown in [10] that this formalism provides much more expressive power than the Possibly and Definitely modalities, and a mapping from $\Re$ to the Possibly and Definitely modalities was given. A conjunctive predicate is of the form $\bigwedge_{i} \phi_{i}$, where $\phi_{i}$ is any predicate defined on variables local to process $P_{i}$. We show that for a conjunctive predicate $\phi$ (e.g., $x_{i}=2 \wedge y_{j}>8$ ), $\operatorname{Possibly}(\phi)$ and Definitely $(\phi)$ can be detected along with the added information of the exact interaction type between each pair of intervals, one interval at each process. This provides flexibility and power to monitor, synchronize, and control distributed executions. The time, space, and message complexities of the 
Table 1. Comparison of space, message and time complexities. $n=$ number of processes, $M=$ maximum queue length at $P_{0}, p=$ maximum number of intervals occurring at any process, $m_{s}=$ total number of messages exchanged between all the processes. Note: $p \geq M$, as all the intervals may not be sent to $P_{0}$.

\begin{tabular}{|c|c|c|c|c|}
\hline & $\begin{array}{c}\text { Avg. time comp- } \\
\text { lexity at } P_{0}\end{array}$ & $\begin{array}{c}\text { Total number } \\
\text { of messages }\end{array}$ & $\begin{array}{c}\text { Space at } P_{0}(= \\
\text { total msg. space })\end{array}$ & $\begin{array}{c}\text { Avg. space at } \\
P_{i}, i \in[1, n]\end{array}$ \\
\hline $\begin{array}{c}\text { GW94 [6] } \\
\text { (Possibly) }\end{array}$ & $O\left(n^{2} M\right)$ or $O\left(n m_{s}\right)$ & $O\left(m_{s}\right)$ & $O\left(n^{2} M\right)$ or $O\left(n m_{s}\right)$ & $O(n)$ \\
\hline $\begin{array}{c}\text { GW96 [7] } \\
\text { (Definitely) }\end{array}$ & $O\left(n^{2} M\right)$ or $O\left(n m_{s}\right)$ & $O\left(m_{s}\right)$ & $O\left(n^{2} M\right)$ or $O\left(n m_{s}\right)$ & $O(n)$ \\
\hline $\begin{array}{c}\text { Fine_Poss, } \\
\text { Fine_Def }, \\
\text { Fine_Rel }\end{array}$ & $O\left(n\left[\min ^{2}\left(4 m_{s}, n p\right)\right]\right)$ & $O\left(\min \left(4 m_{s}, n p\right)\right)$ & $O(\min [(4 n-2) n p$, & $O(n)$ \\
$\left.\left.10 n m_{s}\right]\right)$ & \\
\hline
\end{tabular}

proposed on-line, centralized detection algorithms (Algorithms Fine_Poss and Fine_Def - the main results) to detect Possibly and Definitely in terms of the fine-grained modalities per pair of processes, are the same as those of the earlier on-line, centralized algorithms [6] that can detect only whether the Possibly and Definitely modalities hold. Table 1 compares the complexities. Fine_Rel, which is an intermediate problem we need solve, is introduced later.

The power of our approach stems from the use of intervals as opposed to individual events in the distributed execution. The intervals at each process are identified to be the durations during which the local predicate is true [1012]. We now state Problems Fine_Poss and Fine_Def.

Problem Fine_Poss Statement. For a conjunctive predicate $\phi$, determine online if $\operatorname{Possibly}(\phi)$ is true. If true, identify the fine-grained pairwise interaction between each pair of processes when Possibly $(\phi)$ first becomes true.

Problem Fine_Def Statement. For a conjunctive predicate $\phi$, determine online if Definitely $(\phi)$ is true. If true, identify the fine-grained pairwise interaction between each pair of processes when Definitely $(\phi)$ first becomes true.

Section 2 gives the background and objectives. Section 3 presents the framework and data structures. Section 4 and Section 5 present the on-line algorithms. Section 6 gives the conclusions.

\section{System Model, Background, and Objectives}

\subsection{System Model}

We assume an asynchronous distributed system in which $n$ processes communicate only by reliable message passing. We do not assume FIFO channels. To model the system execution, let $\prec$ be an irreflexive partial ordering representing the causality relation on the event set $E$. $E$ is partitioned into local executions at each process. Let $N$ denote the set of all processes. Each $E_{i}$ is a linearly ordered set of events executed by process $P_{i}$. An event $e$ at $P_{i}$ is denoted $e_{i}$. The causality 
Table 2. Dependent relations for interactions between intervals are given in the first two columns 9. Tests for the relations are given in the third column [10.

\begin{tabular}{|c|c|c|}
\hline Relation $r$ & Expression for $r(X, Y)$ & Test for $r(X, Y)$ \\
\hline R1 & $\forall x \in X \forall y \in Y, x \prec y$ & $V_{y}^{-}[x]>V_{x}^{+}[x]$ \\
\hline $\mathrm{R} 2$ & $\forall x \in X \exists y \in Y, x \prec y$ & $V_{y}^{+}[x]>V_{x}^{+}[x]$ \\
\hline R3 & $\exists x \in X \forall y \in Y, x \prec y$ & $V_{y}^{-}[x]>V_{x}^{-}[x]$ \\
\hline $\mathrm{R} 4$ & $\exists x \in X \exists y \in Y, x \prec y$ & $V_{y}^{+}[x]>V_{x}^{-}[x]$ \\
\hline S1 & $\exists x \in X \forall y \in Y, x \npreceq y \bigwedge y \npreceq x$ & $\begin{array}{c}\text { if } V_{y}^{-}[y] \nless V_{x}^{-}[y] \wedge V_{y}^{+}[x] \ngtr V_{x}^{+}[x] \\
\text { then }\left(\exists x^{0} \in X: V_{y}^{-}[y] \not V_{x}^{x^{0}}[y] \wedge\right. \\
\left.V_{x}^{x^{0}}[x] \not V_{y}^{+}[x]\right) \text { else false }\end{array}$ \\
\hline S2 & $\exists x_{1}, x_{2} \in X \exists y \in Y, x_{1} \prec y \prec x_{2}$ & $\begin{array}{c}\text { if } V_{y}^{+}[x]>V_{x}^{-}[x] \bigwedge V_{y}^{-}[y]<V_{x}^{+}[y] \\
\text { then }\left(\exists y^{0} \in Y: V_{x}^{+}[y] \nless V_{y}^{y^{0}}[y] \wedge\right. \\
\left.V_{y}^{y^{0}}[x] \nless V_{x}^{-}[x]\right) \text { else false }\end{array}$ \\
\hline
\end{tabular}

relation on $E$ is the transitive closure of the local ordering relation on each $E_{i}$ and the ordering imposed by message send events and message receive events [13]. A cut $C$ is a subset of $E$ such that if $e_{i} \in C$ then $\left(\forall e_{i}^{\prime}\right) e_{i}^{\prime} \prec e_{i} \Longrightarrow e_{i}^{\prime} \in C$. A consistent cut is a downward-closed subset of $E$ in $(E, \prec)$ and denotes an execution prefix. For event $e$, there are two special consistent cuts $\downarrow e$ and $e \uparrow . \downarrow e$ is the maximal set of events that happen before $e . e \uparrow$ is the set of all events up to and including the earliest events at each process for which $e$ happens before the events.

Definition 1. Cut $\downarrow e$ is defined to be $\left\{e^{\prime} \mid e^{\prime} \prec e\right\}$ and cut $e \uparrow$ is defined to be $\left\{e^{\prime} \mid e^{\prime} \nsucceq e\right\} \bigcup\left\{e_{i}, i=1, \ldots,|N| \mid e_{i} \succeq e \bigwedge\left(\forall e_{i}^{\prime} \prec e_{i}, e_{i}^{\prime} \nsucceq e\right)\right\}$.

The system state after the events in a cut is a global state; if the cut is consistent, the corresponding system state is a consistent global state. We assume that the popular vector clocks are available [5 15] - the vector clock $V$ has the property that $e \prec f \Longleftrightarrow V(e)<V(f)$.

A conjunctive predicate is of the form $\bigwedge_{i} \phi_{i}$, where $\phi_{i}$ is a predicate defined on variables local to process $P_{i}$. The intervals of interest at each process are the durations in which the local predicate is true. Such an interval at process $P_{i}$ is identified by the (totally ordered) subset of adjacent events of $E_{i}$ for which the local predicate is true.

\subsection{Pairwise Interactions}

There are 29 or 40 possible mutually orthogonal ways in which any two durations can be related to each other, depending on whether the dense or the nondense time model is assumed [9. Informally speaking, with dense time, $\forall x, y$ in interval $A, x \prec y \Longrightarrow \exists z \in A \mid x \prec z \prec y$. These orthogonal interaction types were identified by first using the six relations given in the first two columns of Table 2 Relations R1 (strong precedence), R2 (partially strong precedence), R3 (partially weak precedence), R4 (weak precedence) define causality conditions 
Table 3. The 40 independent relations in $\Re[9$. $X$ and $Y$ are intervals. The upper part of the table gives the 29 relations assuming dense time. The lower part of the table gives 11 additional relations if nondense time is assumed.

\begin{tabular}{|c||c|c|c|c|c|c|c||c|c|c|c|c|c|c||}
\hline \multirow{2}{*}{$\begin{array}{c}\text { Interaction } \\
\text { Type }\end{array}$} & \multicolumn{1}{|c|}{ Relation $r(X, Y)$} & \multicolumn{3}{|c|}{ Relation $r(Y, X)$} \\
\cline { 2 - 10 } & R1 & $\mathrm{R} 2$ & $\mathrm{R} 3$ & $\mathrm{R} 4$ & $\mathrm{~S} 1$ & $\mathrm{~S} 2$ & $\mathrm{R} 1$ & $\mathrm{R} 2$ & $\mathrm{R} 3$ & $\mathrm{R} 4$ & $\mathrm{~S} 1$ & $\mathrm{~S} 2$ \\
\hline \hline$I A\left(=I Q^{-1}\right)$ & 1 & 1 & 1 & 1 & 0 & 0 & 0 & 0 & 0 & 0 & 0 & 0 \\
\hline$I B\left(=I R^{-1}\right)$ & 0 & 1 & 1 & 1 & 0 & 0 & 0 & 0 & 0 & 0 & 0 & 0 \\
\hline$I C\left(=I V^{-1}\right)$ & 0 & 0 & 1 & 1 & 1 & 0 & 0 & 0 & 0 & 0 & 0 & 0 \\
\hline$I D\left(=I X^{-1}\right)$ & 0 & 0 & 1 & 1 & 1 & 1 & 0 & 1 & 0 & 1 & 0 & 0 \\
\hline$I D^{\prime}\left(=I U^{-1}\right)$ & 0 & 0 & 1 & 1 & 0 & 1 & 0 & 1 & 0 & 1 & 0 & 1 \\
\hline$I E\left(=I W^{-1}\right)$ & 0 & 0 & 1 & 1 & 1 & 1 & 0 & 0 & 0 & 1 & 0 & 0 \\
\hline$I E^{\prime}\left(=I T^{-1}\right)$ & 0 & 0 & 1 & 1 & 0 & 1 & 0 & 0 & 0 & 1 & 0 & 1 \\
\hline$I F\left(=I S^{-1}\right)$ & 0 & 1 & 1 & 1 & 0 & 1 & 0 & 0 & 0 & 1 & 0 & 1 \\
\hline$I G\left(=I G^{-1}\right)$ & 0 & 0 & 0 & 0 & 1 & 0 & 0 & 0 & 0 & 0 & 1 & 0 \\
\hline$I H\left(=I K^{-1}\right)$ & 0 & 0 & 0 & 1 & 1 & 0 & 0 & 0 & 0 & 0 & 1 & 0 \\
\hline$I I\left(=I J^{-1}\right)$ & 0 & 1 & 0 & 1 & 0 & 0 & 0 & 0 & 0 & 0 & 1 & 0 \\
\hline$I L\left(=I O^{-1}\right)$ & 0 & 0 & 0 & 1 & 1 & 1 & 0 & 1 & 0 & 1 & 0 & 0 \\
\hline$I L^{\prime}\left(=I P^{-1}\right)$ & 0 & 0 & 0 & 1 & 0 & 1 & 0 & 1 & 0 & 1 & 0 & 1 \\
\hline$I M\left(=I M^{-1}\right)$ & 0 & 0 & 0 & 1 & 1 & 0 & 0 & 0 & 0 & 1 & 1 & 0 \\
\hline$I N\left(=I M^{\prime-1}\right)$ & 0 & 0 & 0 & 1 & 1 & 1 & 0 & 0 & 0 & 1 & 0 & 0 \\
\hline$I N^{\prime}\left(=I N^{\prime-1}\right)$ & 0 & 0 & 0 & 1 & 0 & 1 & 0 & 0 & 0 & 1 & 0 & 1 \\
\hline \hline$I D^{\prime \prime}\left(=(I U X)^{-1}\right)$ & 0 & 0 & 1 & 1 & 0 & 1 & 0 & 1 & 0 & 1 & 0 & 0 \\
\hline$I E^{\prime \prime}\left(=(I T W)^{-1}\right)$ & 0 & 0 & 1 & 1 & 0 & 1 & 0 & 0 & 0 & 1 & 0 & 0 \\
\hline$I L^{\prime \prime}\left(=(I O P)^{-1}\right)$ & 0 & 0 & 0 & 1 & 0 & 1 & 0 & 1 & 0 & 1 & 0 & 0 \\
\hline$I M^{\prime \prime}\left(=\left(I M N^{-1}\right)\right.$ & 0 & 0 & 0 & 1 & 0 & 0 & 0 & 0 & 0 & 1 & 1 & 0 \\
\hline$I N^{\prime \prime}\left(=\left(I M N^{\prime}\right)^{-1}\right)$ & 0 & 0 & 0 & 1 & 0 & 1 & 0 & 0 & 0 & 1 & 0 & 0 \\
\hline$I M N^{\prime \prime}\left(=\left(I M N^{\prime \prime}\right)^{-1}\right)$ & 0 & 0 & 0 & 1 & 0 & 0 & 0 & 0 & 0 & 1 & 0 & 0 \\
\hline
\end{tabular}

whereas S1 and S2 define coupling conditions. Assuming that time is dense, it was shown in 9 , that there are 29 possible interaction types between a pair of intervals, as given in the upper part of Table 3. Of the 29 interactions, there are 13 pairs of inverses, while three are inverses of themselves. The twenty-nine interaction types are specified using boolean vectors. The six relations R1-R4 and S1-S2 form a boolean vector of length 12, (six bits for $r(X, Y)$ and six bits for $r(Y, X))$. The interaction types are illustrated in $[9$. The nondense time model, whose importance is given in [9], permits 11 interaction types between a pair of intervals, defined in the lower part of Table 3, besides the 29 identified before. Of these, there are five pairs of inverses, while one is its own inverse. These interaction types are illustrated in 9 . The set of 40 relations is denoted as $\Re$.

\subsection{Modalities for Global Predicates}

Observe that for any predicate $\phi$, three orthogonal relational possibilities hold under the Possibly/ Definitely classification: (i) Definitely $(\phi)$, (ii) $\neg$ Definitely$(\phi) \wedge \operatorname{Possibly}(\phi)$, (iii) $\neg$ Possibly $(\phi)$. 
Table 4. Refinement mapping [10. The upper part shows the 29 mappings when the dense time model is assumed. With the nondense time model, the 11 additional mappings in the lower part also apply.

\begin{tabular}{|c|c|c|}
\hline Definitely $(\phi)$ & Possibly $(\phi) \wedge \neg$ Definitely $(\phi)$ & $\neg$ Possibly $(\phi)$ \\
\hline \hline ID and IX & IB and IR & IA and IQ \\
ID' and IU & IC and IV & \\
IE and IW & IG & \\
IE' and IT & IH and IK & \\
IF and IS & II and IJ & \\
IO and IL & \\
IP and IL' & & \\
IM & & \\
$\mathrm{IM}^{\prime}$ and IN & & \\
IN & & \\
\hline $\mathrm{ID}^{\prime \prime}$ and IUX & $\mathrm{IM}^{\prime \prime}$ and IMN & \\
$\mathrm{IE}^{\prime \prime}$ and ITW & $\mathrm{IMN}^{\prime \prime}$ & \\
$\mathrm{IL}^{\prime \prime}$ and IOP & & \\
$\mathrm{IN}^{\prime \prime}$ and IMN & & \\
\hline
\end{tabular}

Conjunctive predicates form an important class of predicates and have been studied extensively $[26 / 7 / 8]$. Based on the definitions of the orthogonal temporal interactions [9], the 3 orthogonal relational possibilities based on the Possibly/Definitely definitions were refined into the exhaustive set of 40 possibilities [10]. Table 4 shows this refinement mapping, assuming that the conjunctive predicate is defined on two processes. When conjunctive predicate $\phi$ is defined over variables that are local to $n>2$ processes, one can still express the three possibilities (i) Definitely $(\phi)$, (ii) $\neg \operatorname{Definitely}(\phi) \wedge \operatorname{Possibly}(\phi)$, and (iii) $\neg$ Possibly $(\phi)$, in terms of the fine-grained 40 independent relations between $C_{2}^{n}$ pairs of intervals. Note that not all $40^{C_{2}^{n}}$ combinations will be valid - the combinations have to satisfy the axiom system given in 9 .

For $n>2$ processes, the refinement mappings of the Possibly and Definitely modalities are given by Theorem 10 10].

Theorem 1. [10] Consider a conjunctive predicate $\phi=\wedge_{i} \phi_{i}$. The following results are implicitly qualified over a set of intervals, containing one interval per process.

- Definitely $(\phi)$ holds if and only if $\bigwedge_{(\forall i \in N)(\forall j \in N)}\left[\right.$ Definitely $\left.\left(\phi_{i} \wedge \phi_{j}\right)\right]$

$-\neg$ Definitely $(\phi) \wedge$ Possibly $(\phi)$ holds if and only if

- $(\exists i \in N)(\exists j \in N) \neg$ Definitely $\left(\phi_{i} \wedge \phi_{j}\right) \wedge\left(\bigwedge_{(\forall i \in N)(\forall j \in N)}\left[\operatorname{Possibly}\left(\phi_{i} \wedge\right.\right.\right.$ $\left.\left.\left.\phi_{j}\right)\right]\right)$

- $\neg$ Possibly $(\phi)$ holds if and only if $(\exists i \in N)(\exists j \in N) \neg \operatorname{Possibly}\left(\phi_{i} \wedge \phi_{j}\right)$

Consider the following example (from [10]) of the extra information provided by the fine-grained modalities. Let $\phi$ be $a_{i}=2 \wedge b_{j}=3 \wedge c_{k}=5$. Let $a_{i}, b_{j}$, and $c_{k}$ be 2, 3, 5 respectively, in intervals $X_{i}, Y_{j}$, and $Z_{k}$, respectively, and 


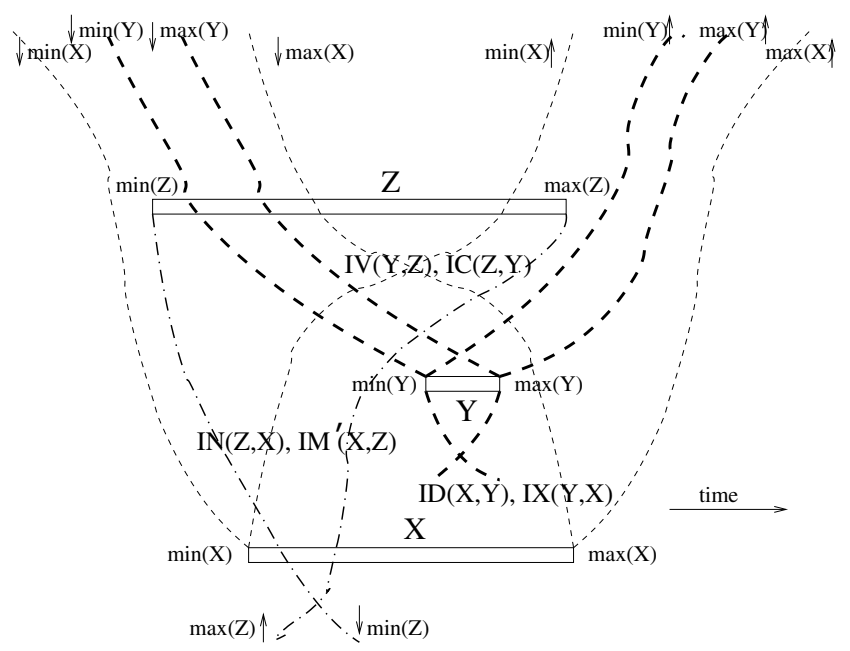

Fig. 1. Example [10] to show fine-grained relations across $n>2$ processes.

let $I D\left(X_{i}, Y_{j}\right), I V\left(Y_{j}, Z_{k}\right)$, and $I N\left(Z_{k}, X_{i}\right)$ be true. This is shown in Figure 1 . Then by Theorem 1, we have (i) Definitely $\left(a_{i}=2 \wedge b_{j}=3\right)$, (ii) Possibly $\left(b_{j}=\right.$ $\left.3 \wedge c_{k}=5\right)$ and $\neg$ Definitely $\left(b_{j}=3 \wedge c_{k}=5\right)$, and (iii) Definitely $\left(a_{i}=\right.$ $\left.2 \wedge c_{k}=5\right)$. By Theorem 1 we have the modality Possibly $(\phi) \wedge \neg D$ Definitely $(\phi)$. Conversely, if Possibly $(\phi) \wedge \neg$ Definitely $(\phi)$ is known in the classical coursegrained classification, the fine-grained classification gives the added information: $I D\left(X_{i}, Y_{j}\right), I V\left(Y_{j}, Z_{k}\right)$, and $I N\left(Z_{k}, X_{i}\right)$.

\subsection{Objective}

Our objective is to solve Fine_Poss and Fine_Def, i.e., to detect Possibly $(\phi)$ and Definitely $(\phi)$, for conjunctive predicates, with the added information of the exact interaction type between each pair of intervals (one at each process) when Possibly $(\phi)$ and Definitely $(\phi)$ are true. The extra information about the pairwise interaction type is useful, as shown in [10] by considering various applications. Another use of the extra information is in multi-player distributed games. The overheads of our algorithms are the same as those of the earlier algorithms, [GW94] [6] for Possibly $(\phi)$ and [GW96] 7] for Definitely $(\phi)$, that can detect only whether Possibly $(\phi)$ is true and whether Definitely $(\phi)$ is true. Tables 1 compares all the performance metrics.

\section{Detecting Predicates: Framework and Data Structures}

Given a conjunctive predicate, for each pair of intervals belonging to different processes, each of the 29 (40) possible independent relations in the dense (nondense) model of time can be tested for using the bit-patterns for the dependent 
1. When an internal event or send event occurs at process $P_{i}, V_{i}[i]=V_{i}[i]+1$.

2. Every message contains the vector clock and Interval Clock of its send event.

3. When process $P_{i}$ receives a message $m s g$, then $\forall j$ do,

$$
\begin{aligned}
& \text { if }(j==i) \text { then } V_{i}[i]=V_{i}[i]+1, \\
& \text { else } V_{i}[j]=\max \left(V_{i}[j], m s g . V[j]\right) .
\end{aligned}
$$

4. When an interval starts at $P_{i}$ (local predicate $\phi_{i}$ becomes true), $I_{i}[i]=V_{i}[i]$.

5. When process $P_{i}$ receives a message $m s g$, then $\forall j$ do,

$$
I_{i}[j]=\max \left(I_{i}[j], \text { msg. } I[j]\right) .
$$

Fig. 2. The vector clock and Interval Clock.

relations, as given in Table 3 The tests for the relations $R 1, R 2, R 3, R 4, S 1$, and $S 2$ are given in the third column of Table 2 using vector timestamps. Recall that the interval at a process is used to identify the period when some local property (using which the predicate $\phi$ is defined) holds. $V_{i}^{-}$and $V_{i}^{+}$denote the vector timestamps at process $P_{i}$ at the start of an interval and the end of an interval, respectively. $V_{i}^{x}$ denotes the vector timestamp of event $x_{i}$ at $P_{i}$.

The tests in Table 2 can be run by a central server along the lines of the algorithms in 46.7814 . Processes $P_{1}, P_{2}, \ldots, P_{n}$ send the timestamps of their intervals and certain other local information to the server $P_{0}$, which maintains queues $Q_{1}, Q_{2}, \ldots, Q_{n}$ for each of the processes. We require that the central server $P_{0}$ receive the updates from each $P_{i}, 1 \leq i \leq n$, in FIFO order. For each of the problems to be solved, the server runs different algorithms to process the interval information in the queues. We assume that interval $X$ occurs at $P_{i}$ and interval $Y$ occurs at $P_{j}$. For any two intervals $X$ and $X^{\prime}$ that occur at the same process, if $R 1\left(X, X^{\prime}\right)$, then we say that $X$ is a predecessor of $X^{\prime}$ and $X^{\prime}$ is a successor of $X$. Also, there are a maximum of $p$ intervals at any process.

The operations and data structures required by the algorithms to solve Problems Fine_Poss and Fine_Def can be divided into two parts. The first, common to all the algorithms, runs on each of the $n$ processes $P_{1}$ to $P_{n}$, and is given in this section. The second part of each algorithm runs on the central process $P_{0}$ and is presented in later sections.

\subsection{Log Operations}

Each process $P_{i}$, where $1 \leq i \leq n$, maintains the following data structures. (1) $V_{i}$ : array[1..n] of integer. This is the Vector Clock. (2) $I_{i}:$ array[1..n] of integer. This is a Interval Clock which tracks the latest intervals at processes. $I_{i}[j]$ is the timestamp $V_{j}[j]$ when $\phi_{j}$ last became true. (3) $\log _{i}$ : Contains the information to be sent to the central process. Figure 2 shows how to maintain the vector clock and Interval Clock.

To maintain $\log _{i}$, the required data structures and operations are defined in Figure 3. $\log _{i}$ is constructed and sent to the central process using the protocol shown. The central process uses the $\log$ to determine the relationship between two intervals. 


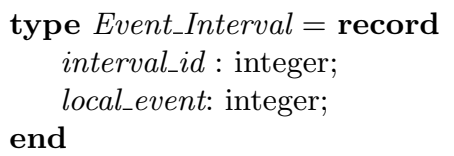

type Process_Log $=$ record

event_interval_queue: queue of Event_Interval; end

Start of an interval:

$\log _{i}$. start $=V_{i}^{-} . \quad$ //Store the timestamp $V_{i}^{-}$of the starting of the interval. On receiving a message during an interval:

if (change in $I_{i}$ ) then //Store local component of vector clock and interval_id for each $k$ such that $I_{i}[k]$ was changed //which caused the change in $I_{i}$ insert $\left(I_{i}[k], V_{i}[i]\right)$ in $\log _{i} . p_{-} \log [k]$.event_interval_queue.

End of interval:

$\log _{i}$.end $=V_{i}^{+} \quad$ //Store the timestamp $V_{i}^{+}$of the end of the interval. if (a receive or send event occurs between start of previous interval and end of current interval) then

Send $\log _{i}$ to central process.

Fig. 3. Data structures and operations to construct $\log$ at $P_{i}(1 \leq i \leq n)$.

\subsection{Complexity Analysis at $P_{i}(1 \leq i \leq n)$}

Space complexity of $\log$. Each $\log$ stores the start $\left(V^{-}\right)$and the end $\left(V^{+}\right)$ of an interval, which requires a maximum of $2 n p$ integers per process. Consider the construction algorithm for $\log$. Besides the start and the end of each interval, an Event_Interval is added to the Log for every component of Interval Clock which is modified due to the receive of a message. As a change in a component of Interval Clock implies the start of a new interval on another process, the total number of times the component of Interval Clock can change is equal to the number of intervals on all the processes. Thus the total number of Event_Interval which can be added to the $\log$ of a single process is $(n-1) p$. This takes $2(n-1) p$ integers per process. The total space needed for Logs corresponding to all $p$ intervals on a single process is $2(n-1) p+2 n p$. This gives an average of $4 n-2$ integers per $\log$. As only one $\log$ exists at a time, the average space requirement at a process $P_{i}(1 \leq i \leq n)$ at any time is the sum of space required by vector clock, Interval Clock, and $L o g$, which is $6 n-2$ integers.

\section{Message complexity of control messages sent to the central process $\boldsymbol{P}_{\mathbf{0}}$} by processes $P_{1}$ to $P_{n}$. This can be determined in two ways. As one message is sent per interval, the number of messages is $O(p)$ for each $P_{i}(i \neq 0)$. This gives a total message complexity of $O(n p)$. On the average, the size of each message is $4 n-2$ as each message contains the $\log$. The total message space overhead for a particular process is the sum of all the $\log$ s for that process, which was shown to be $4 n p-2 p$. Hence the total message space complexity is $4 n^{2} p-2 n p=O\left(n^{2} p\right)$. 
An optimization of message size. The $\log$ corresponding to an interval is sent to the central process only if the relationship between the interval and all other intervals (at other processes) is different from the relationship which its predecessor interval had with all the other intervals (at other processes). Two successive intervals $Y$ and $Y^{\prime}$ on process $P_{j}$ will have the same relationship if no message is sent or received by $P_{j}$ between the start of $Y$ and the end of $Y^{\prime}$. For each message exchanged between processes, a maximum of four interval Logs need to be sent to the central process, because two successive intervals $(Y$ and $Y^{\prime}$ ) will have different relationships if a receive or a send occurs between the start of $Y$ and end of $Y^{\prime}$. This makes it necessary to send two interval Logs for a send event and two for a receive event. Hence if there are $m_{s}$ number of messages exchanged between all processes, then a total of $4 m_{s}$ intervals need to be sent to the central process in $4 m_{s}$ control messages, while the total message space overhead is $2 m_{s} \cdot n+4 m_{s} \cdot 2 n=10 m_{s} n$. The term $2 m_{s} . n$ arises because for every message sent, each other process eventually (due to transitive propagation of Interval Clock) may need to insert a Event_Interval tuple in its Log. This can generate $2 n m_{s}$ overhead in $\operatorname{Logs}$ across the $n$ processes. The term $4 m_{s} .2 n$ arises because the vector clock at the start and end of each interval is sent with each message.

Hence, the total number of control messages sent to the central process and the total message space overhead is the lesser of when either four intervals are sent for each message exchanged or when all the intervals are sent. Thus the total number of messages sent is $O\left(\min \left(4 m_{s}, n p\right)\right)$ and the total message space overhead is $O\left(\min \left(4 n^{2} p-2 n p, 10 m_{s} n\right)\right)$.

\section{Algorithm Fine_Rel: Detecting Fine-Grained Relations}

To solve Problems Fine_Poss and Fine_Def, we first state and solve an intermediate problem.

Problem Fine_Rel Statement: Given a relation $r_{i, j}$ from $\Re$ for each pair of processes $P_{i}$ and $P_{j}$, determine on-line the intervals (if they exist), one from each process, such that each relation $r_{i, j}$ is satisfied by the $\left(P_{i}, P_{j}\right)$ pair.

Note that the given relations $\left\{r_{i, j}, \forall i, j\right\}$ need to satisfy the axioms on $\Re$ [9] for a solution to potentially exist. A distributed and more complex method to solve Fine_Rel, using the data structures of Figure 3 and Theorem 2 below, without proofs or a complexity analysis, is presented in 3.

Algorithm Overview: The algorithm detects a set of intervals, one on each process, such that each pair of intervals satisfies the relationship specified for that pair of processes. If no such set of intervals exists, the algorithm does not return any interval set. The central process $P_{0}$ maintains $n$ queues, one for Logs from each process and determines which orthogonal relation holds between pairs of intervals. The queues are processed using "pruning", described later. If there exists an interval at the head of each queue and these intervals cannot be pruned, then these intervals satisfy $r_{i, j} \forall i, j$, where $i \neq j$ and $1 \leq i, j \leq n$, and hence these intervals form a solution set. 
For $S 2(X, Y)$

1. // Eliminate from $\log$ of interval $Y$ (on $P_{j}$ ), all receives of messages //which were sent by $P_{i}$ before the start of interval $X$ (on $P_{i}$ ).

(1a) for each event_interval $\in \log _{j} . p_{-} \log [i]$. event_interval_queue

(1b) if (event_interval.interval_id $<\log _{i}$. start $\left.[i]\right)$ then delete event_interval.

2. // Select from the pruned $\log$, the earliest message sent from interval $X$ to $Y$.

(2a) temp $=\infty$

(2b) if $\left(\log _{j}\right.$. start $[i] \geq \log _{i}$. start $\left.[i]\right)$ then $\operatorname{temp}=\log _{j} . \operatorname{start}[j]$

(2c) else

(2d) for each event_interval $\in \log _{j} . p_{-} \log [i]$. event_interval_queue temp $=\min ($ temp, event_interval.local_event $)$.

3. if $\left(\log _{i} . e n d[j] \geq t e m p\right)$ then $S 2(X, Y)$ is true.

For $S 1(Y, X)$

1. Same as step 1 of the algorithm to determine $S 2(X, Y)$.

2. Same as step 2 of the algorithm to determine $S 2(X, Y)$.

3. if $\left(\log _{i} . e n d[j]<t e m p\right)$ and $\left(t e m p>\log _{j} . \operatorname{start}[j]\right)$ then $S 1(Y, X)$ is true.

Fig. 4. Tests for coupling relations $S 1(X, Y)$ and $S 2(Y, X)$ at $P_{0}$.

We first define the function $S\left(r_{i, j}\right)$ and the relation $\vdash$. Recall that $X$ and $Y$ are intervals on $P_{i}$ and $P_{j}$, respectively, and $Y^{\prime}$ is any interval that succeeds $Y$.

Definition 2. Function $S: \Re \rightarrow 2^{\Re}$ is defined to be $S\left(r_{i, j}\right)=\{R \in \Re \mid R \neq$ $r_{i, j} \wedge$ if $R(X, Y)$ is true then $r_{i, j}\left(X, Y^{\prime}\right)$ is false for all $Y^{\prime}$ that succeed $\left.Y\right\}$.

Intuitively, for each $r_{i, j} \in \Re$, we define a prohibition function $S\left(r_{i, j}\right)$ as the set of all relations $R$ such that if $R(X, Y)$ is true, then $r_{i, j}\left(X, Y^{\prime}\right)$ can never be true for some successor $Y^{\prime}$ of $Y . S\left(r_{i, j}\right)$ is the set of relations that prohibit $r_{i, j}$ from being true in the future.

Two relations $R^{\prime}$ and $R^{\prime \prime}$ in $\Re$ are related by the allows relation $\vdash$ if the occurrence of $R^{\prime}(X, Y)$ does not prohibit $R^{\prime \prime}\left(X, Y^{\prime}\right)$ for some successor $Y^{\prime}$ of $Y$.

Definition 3. $\vdash$ is a relation on $\Re \times \Re$ such that $R^{\prime} \vdash R^{\prime \prime}$ if (1) $R^{\prime} \neq R^{\prime \prime}$, and (2) if $R^{\prime}(X, Y)$ is true then $R^{\prime \prime}\left(X, Y^{\prime}\right)$ can be true for some $Y^{\prime}$ that succeeds $Y$.

For example, $I C \vdash I B$ because (1) $I C \neq I B$ and, (2) if $I C(X, Y)$ is true, then there is a possibility that $I B\left(X, Y^{\prime}\right)$ is also true, where $Y^{\prime}$ succeeds $Y$.

Lemma 1. If $R \in S\left(r_{i, j}\right)$ then $R \nvdash r_{i, j}$ else if $R \notin S\left(r_{i, j}\right)$ then $R \vdash r_{i, j}$.

Proof. If $R \in S\left(r_{i, j}\right)$, using Definition 2, it can be inferred that $r_{i, j}$ is false for all $Y^{\prime}$ that succeed $Y$. This does not satisfy the second part of Definition 3 , 
Table 5. $S\left(r_{i, j}\right)$ for the 40 independent relations in $\Re$. The upper part of the table gives the function $S$ on 29 relations assuming dense time. The lower part of the table gives function $S$ for the 11 additional relations assuming non-dense time.

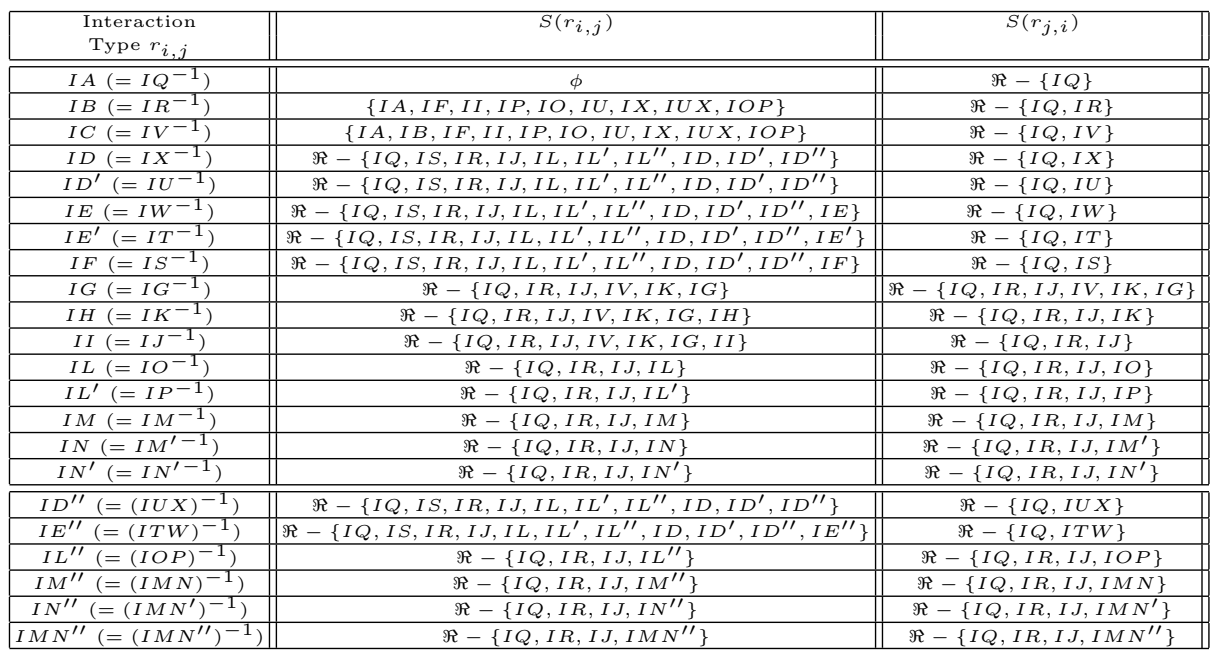

Hence $R \nvdash r_{i, j}$. If $R \notin S\left(r_{i, j}\right)$ and $R \neq r_{i, j}$, it follows that $r_{i, j}$ can be true for some $Y^{\prime}$ that succeeds $Y$. This satisfies Definition 3 and hence $R \vdash r_{i, j}$.

Table 5 gives $S\left(r_{i, j}\right)$ for each of the 40 interaction types in $\Re$. The table is constructed by analyzing each interaction pair in $\Re$. We now state an important result between any two relations in $\Re$ that satisfy the "allows" relation, and the existence of the "allows" relation between their respective inverses. Specifically, if $R^{\prime}$ allows $R^{\prime \prime}$, then Theorem 2 states that $R^{\prime-1}$ necessarily does not allow relation $R^{\prime \prime-1}$. The theorem can be observed to be true from Lemma 1 and Table 5 by a case-by-case analysis.

Theorem 2. For $R^{\prime}, R^{\prime \prime} \in \Re$, if $R^{\prime} \vdash R^{\prime \prime}$ then $R^{\prime-1} \forall R^{\prime \prime-1}$.

Taking the same example, $I C \vdash I B \Rightarrow I V\left(=I C^{-1}\right) \forall I R\left(=I B^{-1}\right)$, which is indeed true. Note that $R^{\prime} \neq R^{\prime \prime}$ in the definition of relation $\vdash$ is necessary; otherwise $R^{\prime} \vdash R^{\prime}$ will become true and from Theorem 2 we get $R^{\prime-1} \forall R^{\prime-1}$ which leads to a contradiction.

Lemma 2. If the relationship $R(X, Y)$ between intervals $X$ and $Y$ (belonging to process $P_{i}$ and $P_{j}$, resp.) is contained in the set $S\left(r_{i, j}\right)$, then interval $X$ can be removed from the queue $Q_{i}$.

Proof. From the definition of $S\left(r_{i, j}\right)$, we get that $r_{i, j}\left(X, Y^{\prime}\right)$ cannot exist, where $Y^{\prime}$ is any successor interval of $Y$. Hence interval $X$ can never be a part of the solution and can be deleted from the queue.

Lemma 3. If the relationship between a pair of intervals $X$ and $Y$ (belonging to processes $P_{i}$ and $P_{j}$ resp.) is not equal to $r_{i, j}$, then either interval $X$ or interval $Y$ is removed from the queue. 
Proof. We use contradiction. Assume relation $R(X, Y)\left(\neq r_{i, j}(X, Y)\right)$ is true for intervals $X$ and $Y$. From Lemma 2, the only time neither $X$ nor $Y$ will be deleted is when $R \notin S\left(r_{i, j}\right)$ and $R^{-1} \notin S\left(r_{j, i}\right)$. From Lemma 1 it can be inferred that $R \vdash r_{i, j}$ and $R^{-1} \vdash r_{j, i}$. As $r_{i, j}^{-1}=r_{j, i}$, we get $R \vdash r_{i, j}$ and $R^{-1} \vdash r_{i, j}^{-1}$. This is a contradiction as by Theorem 2, $R \vdash r_{i, j} \Rightarrow R^{-1} \forall r_{i, j}^{-1}$. Hence $R \in S\left(r_{i, j}\right)$ or $R^{-1} \in S\left(r_{j, i}\right)$ or both, and thus at least one of the intervals can be deleted.

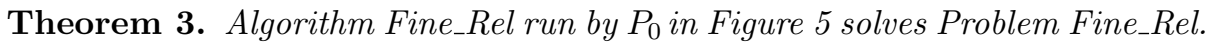

Proof. Lemma 2$]$ which allows queues to be pruned correctly is implemented in the algorithm at $P_{0}$. The algorithm deletes interval $X$ as soon as $R(X, Y) \in$ $S\left(r_{i, j}\right)$ (lines 13-17). Similarly, $Y$ is deleted if $R(Y, X) \in S\left(r_{j, i}\right)$ (lines 15-17). Thus, an interval gets deleted only if it cannot be part of the solution. Also clearly, each interval gets processed unless a solution is found using a predecessor interval from the same process. Lemma 3 gives the unique property that if $R(X, Y) \neq r_{i, j}$, then either interval $X$ or interval $Y$ is deleted. A consequence of this property is that if every queue is non-empty and their heads cannot be pruned, then a solution exists and the set of intervals at the head of each queue forms a solution.

The set updatedQueues stores the indices of all the queues whose heads got updated. In each iteration of the while loop, the indices of all the queues whose head satisfy Lemma 2 are stored in set newUpdatedQueues (lines (13)-(16)). In lines (17) and (18), the heads of all these queues are deleted and indices of the updated queues are stored in the set updatedQueues. Observe that only interval pairs which were not compared earlier are compared in subsequent iterations of the while loop. The loop runs until no more queues can be updated. If at this stage all the queues are non-empty, then a solution is found (follows from Lemma 3). If a solution is found, then for the intervals $X\left(\right.$ at $\left.P_{i}\right)$ and $Y\left(\right.$ at $P_{j}$ ) stored at the heads of these queues, we have $R(X, Y)=r_{i, j}$.

For processes $P_{1}$ to $P_{n}$, the space complexity was shown in Section 3.2 to be on average $O(n)$ at each process. Using the optimization in Section 3.2, the total number of messages sent is equal to $O\left(\min \left(4 m_{s}, p n\right)\right)$ and the total message space complexity is $O\left(\min \left((4 n-2) n p, 10 n m_{s}\right)\right)$.

\section{Theorem 4. Algorithm Fine_Rel has the following complexities.}

- The total message space complexity is $O\left(\min \left((4 n-2) n p, 10 n m_{s}\right)\right)$.

- The total space complexity at process $P_{0}$ is $O\left(\min \left((4 n-2) n p, 10 n m_{s}\right)\right)$.

- The average time complexity at $P_{0}$ is $O\left((n-1) \min \left(4 m_{s}, p n\right)\right)$. This is equivalent to $O\left(n^{2} M\right)$, where $M$ is maximum number of entries in a queue.

Proof. For the central process $P_{0}$, the total space required is $O(\min ((4 n-2) n p,-$ $\left.10 m_{s}\right)$ ) because the total space overhead at $P_{0}$ is equal to the total message space complexity, which was computed in Section 3.2

The time complexity is the product of the number of steps required to determine a relationship and the number of relations determined.

Consider the first part of the product. 
queue of $\log : Q_{1}, Q_{2}, \ldots Q_{n}=\perp$

set of int: updatedQueues, newUpdatedQueues $=\{\}$

On receiving interval from process $P_{z}$ at $P_{0}$

(1) Enqueue the interval onto queue $Q_{z}$

(2) if (number of intervals on $Q_{z}$ is 1) then

(3) updatedQueues $=\{z\}$

(4) while (updatedQueues is not empty) newUpdatedQueues $=\{\}$

for each $i \in$ updatedQueues if $\left(Q_{i}\right.$ is non-empty) then $X=$ head of $Q_{i}$

for $j=1$ to $n$

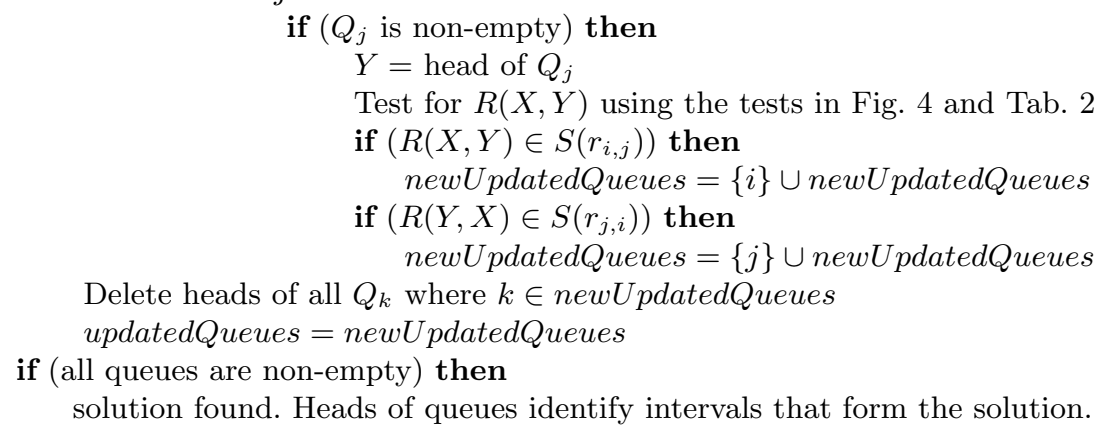

Fig. 5. On-line algorithm Fine_Rel at $P_{0}$.

- The total number of interval pairs between any two processes $P_{i}$ and $P_{j}$ is $p^{2}$. To determine $R 1(X, Y)$ to $R 4(X, Y)$ and $R 1(Y, X)$ to $R 4(Y, X)$, as eight comparisons are needed for each interval pair, a total of $8 p^{2}$ comparisons are necessary for any pair of processes.

- To determine the number of comparisons required by $S 1$ and $S 2$, consider the maximum number of Event_Intervals stored in $\log _{j} . p_{-} \log [i]$ that are sent over the execution lifetime to the central process as part of the Logs. This is the maximum number of Event_Intervals corresponding to $P_{i}$ stored in $Q_{j}$ over $P_{j}$ 's execution lifetime. An Event_Interval is added to $\log _{j} . p_{-} \log [i]$ only when there is a change in the $i^{\text {th }}$ component of Interval Clock at the receive of a message. As the $i^{\text {th }}$ component of Interval Clock changes only when a new interval starts, the total number of times the $i^{\text {th }}$ component of Interval Clock changes is at most equal to $p$, the maximum number of intervals occurring on the other process $P_{i}$. From Figure 4, it can be observed that for each Event_Interval, there is one comparison. Thus, to determine the relationship between an interval on $P_{i}$ and all other intervals on $P_{j}$, the number of comparisons is equal to $p$. As there are $p$ intervals on $P_{i}$, a total of $p^{2}$ comparisons are required to determine $S 1$ or $S 2$. Hence the total number of comparisons to determine $S 1(X, Y), S 2(X, Y), S 1(Y, X)$, and $S 2(Y, X)$ is $4 p^{2}$. 
This gives a total of $8 p^{2}+4 p^{2}=O\left(p^{2}\right)$ comparisons to determine the relation between each pair of intervals on a pair of processes. As there are a total of $p^{2}$ intervals pairs between two processes, the average number of comparisons required to determine a relationship is $O(1)$.

To analyse the second part of the product, consider Figure [5] For each interval considered from one of the queues in updatedQueues (lines (6)-(12)), the number of relations determined is $n-1$. Thus the number of relations determined for each iteration of the while loop is $(n-1) \mid$ updatedQueues $\mid$, where $\mid$ updatedQueues $\mid$ denotes the number of entries in updatedQueues. The cumulative $\sum \mid$ updatedQueues $\mid$ over all iterations of the while loop is less than the total number of intervals over all the queues. Thus, the total number of relations determined is less than $(n-1) \min \left(4 m_{s}, p n\right)$, where $\min \left(4 m_{s}, n p\right)$ is the upper bound on the total number of intervals over all the queues. As the average time required to determine a relationship is $O(1)$, the average time complexity of the algorithm is equal to $O\left((n-1) \min \left(4 m_{s}, p n\right)\right)$.

The average time complexity can be equivalently expressed using $M$, the maximum number of entries in a queue, as follows. The total number of intervals over all the queues is $O(n M)$. As the total number of relations determined is $(n-$ 1) $\sum \mid$ updatedQueues $\mid$ over all the iterations of the while loop, this is equivalent to $(n-1) \cdot n M=O\left(n^{2} M\right)$. This is also the average time complexity because it takes $O(1)$ time on the average to determine a relationship.

Table 1 compares the complexities of Fine_Rel with those of GW94 6] and GW96 7]. GW94 and GW96 computed their time complexity at $P_{0}$ as only $O\left(n^{2} M\right)$, not in terms of $m_{s}$ or $p$. They did not give the space complexity at $P_{0}$. As each control message in GW94 and GW96 carries a fixed size $O(n)$ message overhead and a control message is sent to $P_{0}$ for every message send/receive event, we have computed their total space complexity and average time complexity at $P_{0}$ as $O\left(n m_{s}\right)$. This enables a direct comparison with the complexities of our algorithm. Further, we have also computed our average time complexity using $M$, as $O\left(n^{2} M\right)$. In our algorithm, note that $M \leq p ; M=p$ if the message overhead optimization is not used. We do not express the total space at $P_{0}$ in terms of $M$ because the queue entries are of variable size, with an average size of $(4 n-2)$ integers.

\section{Algorithms Fine_Poss and Fine_Def}

By leveraging Theorem 1 and the mapping of fine-grained modalities to Possibly and Definitely modalities, as given in Table 4, we address the problems of determining whether Possibly $(\phi)$ and Definitely $(\phi)$ hold. If either of these two coarse-grained modalities holds, we can also determine the exact fine-grained orthogonal relation/modality between each pair of processes, unlike any previous algorithm. Further, the time, space, and message complexities of the proposed on-line (centralized) detection algorithms (Algorithms Fine_Poss and Fine_Def) to detect Possibly and Definitely in terms of the fine-grained modalities per pair of processes, are the same as those of the earlier on-line 
(centralized) algorithms 6.7. that can detect only whether the Possibly and Definitely modalities hold.

Recall that $\Re$ is a set of orthogonal relations and hence one and only one relation from $\Re$ must hold between any pair of intervals. Consider the case where, for each pair of processes $\left(P_{i}, P_{j}\right)$, we are given a set $r_{i, j}^{*} \subseteq \Re$ such that we are satisfied if some relation in $r_{i, j}^{*}$ holds. Now consider the objective where we need to identify one interval per process such that for each process pair $\left(P_{i}, P_{j}\right)$, some relation in $r_{i, j}^{*}$ holds for that $\left(P_{i}, P_{j}\right)$. Such an objective would be useful if we can

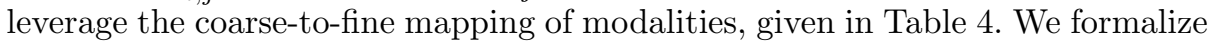
such an objective by generalizing the detection problem Fine_Rel to problem Fine_Rel', as follows.

Problem Fine_Rel' Statement: Given a set of relations $r_{i, j}^{*} \subseteq \Re$ for each pair of processes $P_{i}$ and $P_{j}$, determine on-line the intervals, if they exist, one from each process, such that any one of the relations in $r_{i, j}^{*}$ is satisfied (by the intervals) for each $\left(P_{i}, P_{j}\right)$ pair. If a solution exists, identify the fine-grained interaction from $\Re$ for each pair of processes in the first solution.

To solve Fine_Rel', given an arbitrary $r_{i, j}^{*}$, a solution based on algorithm Fine_Rel (Figure 5) will not work because in the crucial tests in lines (13)-(14), neither interval may be removable, and yet none of the relations from $r_{i, j}^{*}$ might hold between the two intervals. This leads to deadlock! To see this further, let $r 1, r 2 \in r_{i, j}^{*}$ and let $R(X, Y)$ hold, where $R \notin r_{i, j}^{*}$. Now let $R \in S(r 1)$, $R^{-1} \notin S\left(r 1^{-1}\right), R \notin S(r 2), R^{-1} \in S\left(r 2^{-1}\right)$. Interval $X$ cannot be deleted because $r 2\left(X, Y^{\prime}\right)$ may be true for a successor $Y^{\prime}$. Interval $Y$ cannot be deleted because $r 1^{-1}\left(Y, X^{\prime}\right)$ may be true for a successor $X^{\prime}$. Therefore, a solution based on Algorithm Fine_Rel will deadlock, and a more elaborate (and presumably expensive) solution will be needed.

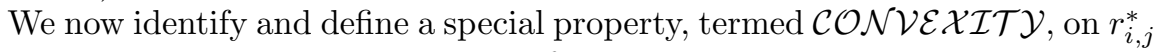
such that the deadlock is prevented. Informally, this property says that there is no relation $R$ outside $r_{i, j}^{*}$ such that for any $r 1, r 2 \in r_{i, j}^{*}, R \vdash r 1$ and $R^{-1} \vdash r 2^{-1}$. This property guarantees that when intervals $X$ and $Y$ are compared for $r_{i, j}^{*}$ and $R(X, Y)$ holds, either $X$ or $Y$ or both get deleted, and hence there is progress. The sets $r_{i, j}^{*}$, derived from Table 4 , that need to be detected to solve Problems Fine_Poss and Fine_Def satisfy this property. We therefore observe that problems Fine_Poss and Fine_Def are special cases of Problem Fine_Rel' in which the property $\mathcal{C O N} \mathcal{V} \mathcal{E} \mathcal{X} \mathcal{T} \mathcal{Y}$ on $r_{i, j}^{*}$ is necessarily satisfied. To solve Problems Fine_Poss and Fine_Def, we then use the generalizations of Lemmas 2 and 3. as given in Lemmas 4 and 5 respectively, to first solve Fine_Rel'.

\section{Definition 4.}

$\mathcal{C O N} \mathcal{V} \mathcal{E} \mathcal{I} \mathcal{T Y}: \forall R \notin r_{i, j}^{*}:\left(\forall r_{i, j} \in r_{i, j}^{*}, R \in S\left(r_{i, j}\right) \bigvee \forall r_{j, i} \in r_{j, i}^{*}, R^{-1} \in S\left(r_{j, i}\right)\right)$

Lemma 4. If the relationship $R(X, Y)$ between intervals $X$ and $Y$ (belonging to processes $P_{i}$ and $P_{j}$, respectively) is contained in the set $\bigcap_{r_{i, j} \in r_{i, j}^{*}} S\left(r_{i, j}\right)$, then interval $X$ can be removed from the queue $Q_{i}$. 


$$
\begin{aligned}
& \text { if }\left(R(X, Y) \in \bigcap_{r_{i, j} \in r_{i, j}^{*}} S\left(r_{i, j}\right)\right) \text { then } \\
& \quad \text { newUpdatedQueues }=\{i\} \cup \text { newUpdatedQueues } \\
& \text { if }\left(R(Y, X) \in \bigcap_{r_{j, i} \in r_{j, i}^{*}} S\left(r_{j, i}\right)\right) \text { then } \\
& \quad \text { newUpdatedQueues }=\{j\} \cup \text { newUpdatedQueues }
\end{aligned}
$$

Fig. 6. Algorithm Fine_Rel': Changes to algorithm Fine_Rel are listed, assuming $r_{i, j}^{*}$ satisfies property $\mathcal{C O} \mathcal{N} \mathcal{E} \mathcal{X} \mathcal{I} \mathcal{T}$.

Proof. From the definition of $S\left(r_{i, j}\right)$, we infer that no relation $r_{i, j}\left(X, Y^{\prime}\right)$, where $r_{i, j} \in r_{i, j}^{*}$ and $Y^{\prime}$ is any successor interval of $Y$ on $P_{j}$, can be true. Hence interval $X$ can never be a part of the solution and can be deleted from the queue.

Lemma 5. If the relationship $R(X, Y)$ between a pair of intervals $X$ and $Y$ (belonging to processes $P_{i}$ and $P_{j}$, respectively) does not belong to the set $r_{i, j}^{*}$, where $r_{i, j}^{*}$ satisfies property $\mathcal{C O N} \mathcal{V} \mathcal{E} \mathcal{X} \mathcal{I} \mathcal{Y}$, then either interval $X$ or interval $Y$ is removed from the queue.

Proof. We use contradiction. Assume relation $R(X, Y)\left(\notin r_{i, j}^{*}(X, Y)\right)$ is true for intervals $X$ and $Y$. From Lemma 4, the only time neither $X$ nor $Y$ will be deleted is when both $R \notin \bigcap_{r_{i, j} \in r_{i, j}^{*}} S\left(r_{i, j}\right)$, and $R^{-1} \notin \bigcap_{r_{j, i} \in r_{j, i}^{*}} S\left(r_{j, i}\right)$. However, as $r_{i, j}^{*}$ satisfies property $\mathcal{C O} \mathcal{N} \mathcal{V} \mathcal{E} \mathcal{X} \mathcal{I} \mathcal{T} \mathcal{Y}$, we have that $R \in \bigcap_{r_{i, j} \in r_{i, j}^{*}} S\left(r_{i, j}\right)$ or $R^{-1} \in \bigcap_{r_{j, i} \in r_{j, i}^{*}} S\left(r_{j, i}\right)$ must be true. Thus at least one of the intervals can be deleted by an application of Lemma 4 .

The proof of the following theorem is similar to the proof of Theorem 3 .

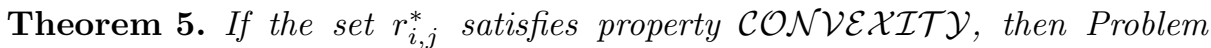
Fine_Rel' is solved by replacing lines (13) and (15) in algorithm Fine_Rel in Figure 5 by the lines (13) and (15) in Figure 6 .

Proof. Analogous to the proof of Theorem 3 . Use Lemmas 4 and 5 instead of Lemmas 2 and 3 respectively, and reason with $r_{i, j}^{*}$ instead of with $r_{i, j}$.

Corollary 1. The time, space, and message complexities of Algorithm Fine Rel' are the same as those of Algorithm Fine_Rel, which were stated in Theorem 4 .

Proof. The only changes to Algorithm Fine_Rel are in lines (13) and (15). In Algorithm Fine_Rel', instead of checking $R(X, Y)$ for membership in $S\left(r_{i, j}\right)$ in line (13), $R(X, Y)$ is checked for membership in $\bigcap_{r_{i, j} \in r_{i, j}^{*}} S\left(r_{i, j}\right)$. Both $S\left(r_{i, j}\right)$ and $\bigcap_{r_{i, j} \in r_{i, j}^{*}} S\left(r_{i, j}\right)$ are sets of size between 0 and 40 . An analogous observation holds for the change on line (15). Hence, the time, space, and message complexities of Fine_Rel are unaffected in Fine_Rel'.

To detect Possibly $(\phi), r_{i, j}^{*}$ is set to the union of the orthogonal interactions in the first two columns of Table 4. We can verify (by case-by-case enumeration) that $r_{i, j}^{*}$ does satisfy property $\mathcal{C O N} \mathcal{V} \mathcal{E} \mathcal{X} \mathcal{I} \mathcal{Y}$. Similarly, to detect 
Definitely $(\phi), r_{i, j}^{*}$ is set to the union of the orthogonal interactions in the first column of Table 4 We can verify (by case-by-case enumeration) that $r_{i, j}^{*}$ does satisfy property $\mathcal{C O N} \mathcal{V} \mathcal{E} \mathcal{X} \mathcal{T} \mathcal{Y}$.

The following two theorems about using algorithm Fine_Rel' (Figure 6) to solve Problems Fine_Poss and Fine_Def can be readily proved by using Theorem 1 the refinement mapping of Table 4, and Theorem [5. The two resulting algorithms are named Fine_Poss and Fine_Def, respectively.

Theorem 6. Algorithm Fine_Rel modified to algorithm Fine_Rel' (Figure 6) solves Problem Fine_Poss (about Possibly $(\phi))$ when $r_{i, j}^{*}$ is set to the union of the relations in the first and second columns of Table 4 .

Proof. From Theorem 1, Possibly $(\phi)$ is true if and only if $(\forall i \in N)(\forall j \in$ $N)$ Possibly $\left(\phi_{i} \wedge \phi_{j}\right)$. For any $i$ and $j$, Possibly $\left(\phi_{i} \wedge \phi_{j}\right)$ is true if and only if $R\left(X_{i}, Y_{j}\right)$ is any of the temporal relations given in the first two columns of Table 4. When $r_{i, j}^{*}$ is set to the union of the relations in these two columns,

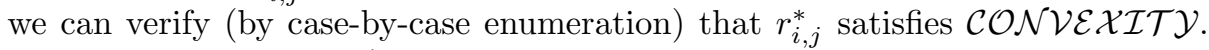
As Algorithm Fine_Rel' is correct (by Theorem [6), when its $r_{i, j}^{*}$ is instantiated with the set above to get Algorithm Fine_Poss, we have that Fine_Poss is also correct.

Theorem 7. Algorithm Fine_Rel modified to algorithm Fine_Rel' (Figure 6) solves Problem Fine_Def (about Definitely $(\phi))$ when $r_{i, j}^{*}$ is set to the union of the relations in the first column of Table 4.

Proof. From Theorem 1, Definitely $(\phi)$ is true if and only if $(\forall i \in N)(\forall j \in$ $N)$ Definitely $\left(\phi_{i} \wedge \phi_{j}\right)$. For any $i$ and $j$, Definitely $\left(\phi_{i} \wedge \phi_{j}\right)$ is true if and only if $R\left(X_{i}, Y_{j}\right)$ is any of the temporal relations given in the first column of Table 4 . When $r_{i, j}^{*}$ is set to the relations in this column, we can verify (by case-by-

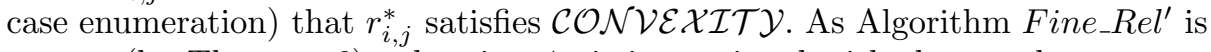
correct (by Theorem [6), when its $r_{i, j}^{*}$ is instantiated with the set above to get Algorithm Fine_Def, we have that Fine_Def is also correct.

In algorithm Fine_Rel', when $r_{i, j}^{*}$ is set to the values as specified in Theorems [6]and[7to detect Possibly and Definitely, respectively, set $\bigcap_{r_{i, j} \in r_{i, j}^{*}} S\left(r_{i, j}\right)$ used in line (13) of the algorithm becomes $\{I A\}$ and $\{I A, I B, I C, I G, I H, I I\}$, respectively. An identical change occurs to the set $\bigcap_{r_{j, i} \in r_{j, i}^{*}} S\left(r_{j, i}\right)$ on line (15).

Corollary 2. The time, space, and message complexities of Algorithms Fine_Poss and Fine_Def are the same as those of Algorithm Fine_Rel (stated in Theorem (4) and of Algorithm Fine_Rel' (stated in Corollary 1).

Proof. Follows from Corollary 1 and the fact that $r_{i, j}^{*}$ for Fine_Poss and Fine_Def satisfy $\mathcal{C O N} \mathcal{V E X} \mathcal{X} \mathcal{T} \mathcal{Y}$ and are instantiations of $r_{i, j}^{*}$ in Fine_Rel'.

\section{Discussion \& Conclusions}

This paper presented algorithms to detect conjunctive predicates under finegrained modalities. Algorithms Fine_Poss and Fine_Def not only detect Poss$i b l y(\phi)$ and Definitely $(\phi)$, respectively, but also (unlike previous algorithms) 
return the pairwise fine-grained relations which exist between all the intervals in the solution set. The space, message, and computational complexities of the previous works for conjunctive predicate detection, GW94 [6] and GW96 [7], for detection of only Possibly $(\phi)$ and Definitely $(\phi)$, respectively, is compared with our algorithms in Table1. All the complexity measures for algorithms Fine_Poss and Fine_Def are the same as those for GW94 [6] and GW96 [7]. Thus with the same overhead, Algorithms Fine_Poss and Fine_Def do the extra work of finding the fine-grained relations which exist between the intervals contained in the solution set for Possibly and Definitely.

A detailed version of these results appears in [1]. Distributed algorithms can be devised for Fine_Poss and Fine_Def based on the distributed algorithm given in [3] to solve Fine_Rel. A discussion of how intervals might be identified when trying to use the fine-grained modalities on nonconjunctive predicates, i.e., general relational predicates, is given in [11.

\section{Acknowledgements}

This material is based upon work supported by the National Science Foundation under Grant No. CCR-9875617.

\section{References}

1. Chandra, P., Kshemkalyani, A.D.: Algorithms for Detecting Global Predicates under Fine-grained Modalities, Technical Report UIC-ECE-02-05, University of Illinois at Chicago, April 2002.

2. Chandra, P., Kshemkalyani, A.D.: Distributed Algorithm to Detect Strong Conjunctive Predicates, Information Processing Letters, 87(5): 243-249, September 2003.

3. Chandra, P., Kshemkalyani, A.D.: Detection of Orthogonal Interval Relations, Proc. High-Performance Computing Conference, 323-333, LNCS 2552, Springer, 2002.

4. Cooper, R., Marzullo, K.: Consistent Detection of Global Predicates, Proc. ACM/ONR Workshop on Parallel \& Distributed Debugging, 163-173, May 1991.

5. Coulouris, G., Dollimore, J., Kindberg, T.: Distributed Systems Concepts and Design, Addison-Wesley, 3rd edition, 2001.

6. Garg, V.K., Waldecker, B.: Detection of Weak Unstable Predicates in Distributed Programs, IEEE Trans. Parallel \& Distributed Systems, 5(3), 299-307, Mar. 1994.

7. Garg, V.K., Waldecker, B.: Detection of Strong Unstable Predicates in Distributed Programs, IEEE Trans. Parallel \& Distributed Systems, 7(12):1323-1333, Dec. 1996.

8. Hurfin, M., Mizuno, M., Raynal, M., Singhal, M.: Efficient Distributed Detection of Conjunctions of Local Predicates, IEEE Trans. Software Engg., 24(8): 664-677, 1998.

9. Kshemkalyani, A.D.: Temporal Interactions of Intervals in Distributed Systems, Journal of Computer and System Sciences, 52(2): 287-298, April 1996.

10. Kshemkalyani, A.D.: A Fine-Grained Modality Classification for Global Predicates, IEEE Trans. Parallel \& Distributed Systems, 14(8): 807-816, August 2003. 
11. Kshemkalyani, A.D.: A Note on Fine-grained Modalities for Nonconjunctive Predicates, 5th Workshop on Distributed Computing, LNCS, Springer, Dec. 2003.

12. Kshemkalyani, A.D.: A Framework for Viewing Atomic Events in Distributed Computations, Theoretical Computer Science, 196(1-2), 45-70, April 1998.

13. Lamport, L.: Time, Clocks, and the Ordering of Events in a Distributed System, Communications of the ACM, 558-565, 21(7), July 1978.

14. Marzullo, K., Neiger, G.: Detection of Global State Predicates, Proc. 5th Workshop on Distributed Algorithms, LNCS 579, Springer-Verlag, 254-272, October 1991.

15. Mullender, S.: Distributed Systems, 2nd Edition, ACM Press, 1994.

16. Stoller, S., Schneider, F.: Faster Possibility Detection by Combining Two Approaches, Proc. 9th Workshop on Distributed Algorithms, 318-332, LNCS 972, Springer-Verlag, 1995.

17. Venkatesan, S., Dathan, B.: Testing and Debugging Distributed Programs Using Global Predicates, IEEE Trans. Software Engg., 21(2), 163-177, Feb. 1995. 